\title{
Hacia el reconocimiento internacional de las publicaciones científicas españolas
}

\author{
María Bordons \\ Centro de Información y Documentación Científica (CINDOC). CSIC. Madrid. España.
}

Las publicaciones científicas desempeñan un papel fundamental en las distintas etapas de la actividad investigadora. Constituyen el punto de partida de cualquier investigación, pues aportan el estado del conocimiento en un tema determinado, y son el canal preferente por que darán a conocer los resultados de una investigación ya concluida. Facilitan la difusión del nuevo conocimiento, que será la base para futuras investigaciones de otros científicos, de forma que las contribuciones de sucesivas generaciones de investigadores hacen posible el crecimiento acumulativo de la ciencia y, en última instancia, el progreso científico. Pero además las publicaciones cumplen otras funciones, como establecer la prioridad de un determinado descubrimiento a través de la fecha de recepción de un artículo en una revista, y recompensar a los investigadores por sus contribuciones a través de las citas. Finalmente, no se puede dejar de mencionar que las publicaciones científicas en las áreas más aplicadas o clínicas constituyen un apoyo esencial para la actualización de conocimientos y para el buen desarrollo de la práctica profesional.

Partiendo de estas premisas, que ponen de manifiesto la importancia de las publicaciones en el proceso investigador, se puede afirmar que los estudios bibliométricos o de producción científica constituyen actualmente un instrumento esencial para el análisis y la evaluación de la investigación desarrollada en los distintos países, complementando con éxito a otro tipo de indicadores, como pueden ser las inversiones económicas o los recursos humanos en investigación. Los estudios sobre publicaciones utilizan con frecuencia la

\section{VÉASE ARTÍCULO EN PÁGS. 806-14}

Correspondencia: Dra. María Bordons.

Centro de Información y Documentación Científica (CINDOC). CSIC.

Joaquín Costa, 22. 28002 Madrid. España.

Correo electrónico: mbordons@cindoc.csic.es

Full English text available at: www.revespcardiol.org
Web of Science, base de datos multidisciplinaria muy adecuada para analizar la ciencia en su vertiente más internacional, ya que cubre una selección de revistas atendiendo a criterios de calidad e interés internacional.

El número de publicaciones de autores españoles en la base de datos Science Citation Index Expanded, accesible a través de la Web of Science, ha aumentado exponencialmente desde 1980 hasta la actualidad. Las publicaciones de España en dicha base de datos se situaban en torno a los 4.000 documentos en 1980 (el $0,7 \%$ del total de la base de datos en dicho año), y ascendían a casi 31.000 documentos en 2003 (el 2,8\% de la producción mundial en ese año), lo que supone un incremento de más del $600 \%$, muy superior al aumento total de la base de datos (100\%) (fig.1). De estas publicaciones, prácticamente el $45 \%$ se refería a temas médicos, denominación que incluye una amplia gama de disciplinas que abarcan investigación básica (biomedicina) y clínica (medicina clínica).

La creciente presencia de los investigadores españoles en las bases de datos bibliográficas internacio-

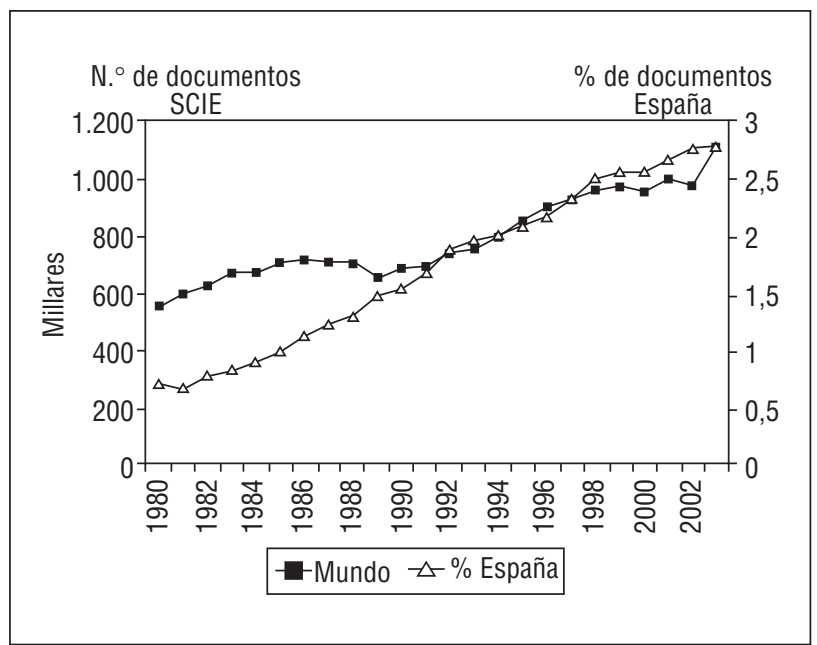

Fig. 1. Evolución anual del número de publicaciones científicas recogidas en el SCI Expanded y porcentaje de España sobre el total. Fuente: Web of Science. 


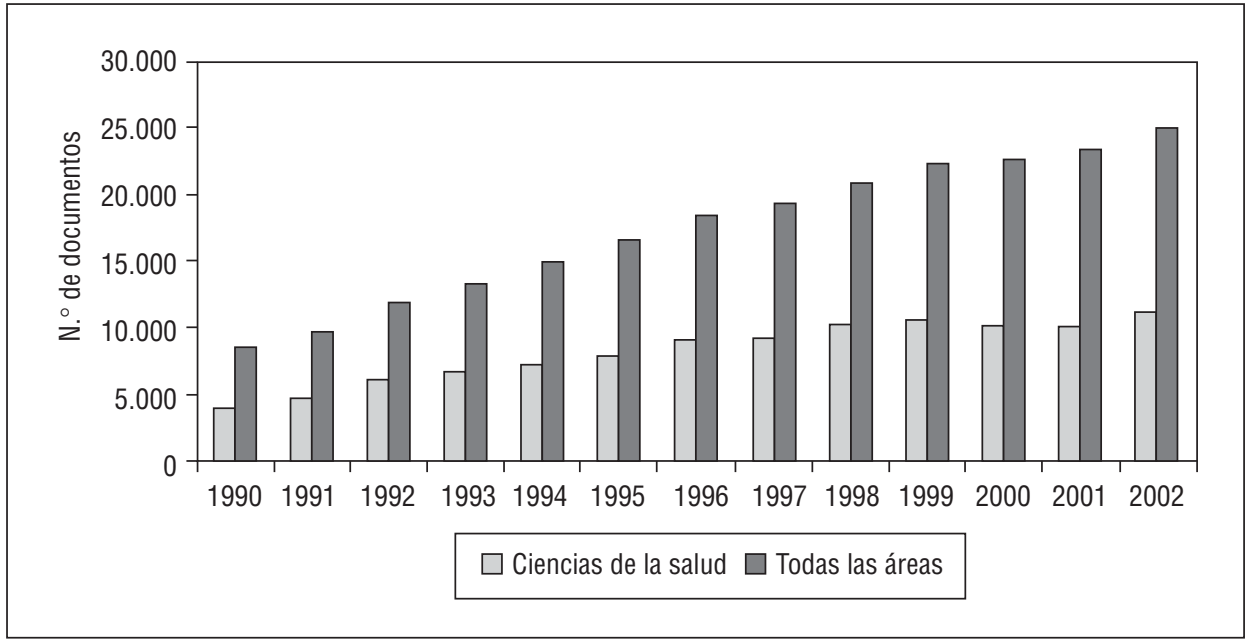

Fig. 2. Publicaciones científicas de España en el SCl, versión CD-ROM, 1990-2002. nales es un hecho positivo que muestra el fortalecimiento del sistema español de investigación y, también, su mayor orientación internacional en las últimas décadas. Esta tendencia responde a diversos factores, algunos que podríamos llamar «intrínsecos», inherentes al proceso científico, y otros «extrínsecos» a éste. Entre los primeros hay que mencionar que la ciencia es, por definición, una empresa internacional y colectiva cuyos avances interesan a toda la comunidad científica, independientemente de su nacionalidad o ubicación geográfica. Conseguir la mayor difusión posible de la propia investigación para hacerla accesible y útil es, pues, un objetivo natural y legítimo; asimismo, publicar en revistas de difusión internacional garantiza una mayor difusión de la investigación. Pero, además de estos factores intrínsecos, hay unos factores extrínsecos, secundarios a las políticas de gestión y evaluación de la investigación y de la práctica profesional, que influyen en los investigadores a la hora de elegir sus revistas de publicación. El mayor reconocimiento otorgado en distintos procesos de evaluación (p. ej., en la concesión de sexenios, ayudas a la investigación, becas o incluso premios) a las publicaciones en revistas científicas internacionales de prestigio es, sin duda alguna, un estímulo eficaz para aumentar la visibilidad internacional de la investigación de un país ${ }^{1}$.

El proceso de internacionalización que afecta hoy día a muchos aspectos de nuestras vidas está también presente en el entorno científico. Las nuevas tecnologías de la información facilitan no sólo el acceso al nuevo conocimiento en cualquier parte del mundo, sino también la comunicación entre los investigadores. Como resultado, se observa una creciente colaboración internacional entre investigadores, la formación de redes y el creciente uso de canales internacionales para la difusión de la propia investigación. Así, en muchos países avanzados se ha observado en los últimos años un cambio en las estrategias de publicación de los investigadores: pasan de publicar en revistas nacionales a hacerlo en las internacionales, con el fin de obtener una mayor difusión y visibilidad de su investigación. Esta transición parece producirse de forma paulatina y se han descrito 3 etapas consecutivas ${ }^{2,3}$ :

- En la primera etapa, los autores orientan sus publicaciones hacia revistas internacionales, recogidas en las principales bases de datos de sus especialidades ( $p$. ej., SCI o MEDLINE), lo que garantiza una amplia visibilidad de sus trabajos.

- La segunda etapa conlleva una mayor selectividad en las revistas de publicación elegidas: se observa la preferencia por revistas en lengua inglesa, para las que se ha descrito mayor difusión y mayor reconocimiento a través de citas.

- Finalmente, el objetivo de la tercera etapa es publicar en las revistas de más calidad y prestigio de entre todas las internacionales. Aunque es difícil evaluar la calidad científica de las revistas, uno de los indicadores más utilizados es su factor de impacto, calculado y publicado anualmente por el Institute for Scientific Information (ISI) de Filadelfia en el Journal Citation Reports. Publicar en revistas con un alto factor de impacto se convierte, así, en un objetivo para muchos investigadores.

Los datos disponibles sobre España muestran claramente esta transición. Desde 1980 hasta 1999, las publicaciones científicas de España en el Science Citation Index Expanded se multiplicaron por un factor de 8. Si nos referimos a las ciencias biomédicas y de la salud, la producción de España en el período 1990-1999 (SCI restringido, versión CD-ROM) se multiplicó por 3, crecimiento muy similar al observado para el total del país en dicho período (2,8 frente a 2,9) (fig. 2). El aumento experimentado en los últimos años por la producción en revistas internacionales ha sido superior al observado en las revistas nacionales recogidas por las bases de datos españolas IME (Índice Médico 
Español) e ICYT (Índice Español de Ciencia y Tecnología), y se ha llegado a observar incluso una tendencia negativa en el último caso. Se pone así de manifiesto una clara orientación de los investigadores hacia las publicaciones internacionales (etapa 1), impulsada probablemente por una mezcla de los factores intrínsecos y extrínsecos que comentábamos al principio.

La migración hacia revistas en lengua inglesa también se ha puesto de manifiesto en nuestro país. La tabla 1 muestra el descenso del número de publicaciones en español recogidas en el SCI desde 1980 hasta 2000. Esta tendencia se ha observado también en otros idiomas, como el francés o el alemán, y se acompaña de un incremento en el idioma inglés, en el que estaba escrito el $96 \%$ de los documentos recogidos por el SCI Expanded en 2000. Se confirma, pues, la etapa 2 de la transición.

La etapa 3 conlleva mayor dificultad. Pero los datos disponibles muestran que los investigadores españoles tienden a publicar en revistas con un factor de impacto relativamente alto, con variaciones según las disciplinas. En concreto, analizando las publicaciones de autores españoles en el área de biomedicina y ciencias de la salud, se observa una tendencia a publicar en las revistas situadas en el primer y segundo cuartil de cada disciplina, consideradas las revistas en orden descendente de factor de impacto dentro de cada disciplina ${ }^{4}$.

La internacionalización no se produce al mismo ritmo en todas las áreas y disciplinas. Tiene su mayor sentido en las áreas más básicas de investigación, en las que hay una comunidad científica internacional que comparte objetivos y preocupaciones; son áreas en las que el inglés se ha impuesto como lingua franca para facilitar la comunicación más allá de fronteras geográficas y lingüísticas. Aunque la internacionalización también alcanza a las áreas clínicas de investigación, su instauración en éstas es más suave, ya que con frecuencia coexisten intereses nacionales e internacionales, no siempre coincidentes.

El trabajo de Miguel-Dasit et $a l^{5}$ publicado en este número de la Revista analiza la visibilidad internacional de las publicaciones de los cardiólogos y radiólogos españoles en un área clínica: diagnóstico por imagen en cardiología. No cabe duda de que ésta es un área de gran interés y con gran potencial futura por el progresivo papel de estas técnicas en el diagnóstico cardiovascular ${ }^{6}$. Entre los resultados del estudio de Miguel-Dasit et al, podríamos destacar 2 especialmente interesantes: la creciente visibilidad internacional del área y el importante papel de las revistas de las sociedades científicas de ambas especialidades.

Atendiendo al modelo de internacionalización previamente expuesto, el trabajo de Miguel-Dasit et al sugiere que la incorporación de los radiólogos a la comunidad científica internacional ha sido posterior a la de los cardiólogos. Se observa una evolución ascen-
TABLA 1. Distribución anual de publicaciones por idioma (Science Citation Index Expanded, Web of Science)

\begin{tabular}{lrrr}
\hline & $\mathbf{1 9 8 0}$ & $\mathbf{1 9 9 0}$ & \multicolumn{1}{c}{$\mathbf{2 0 0 0}$} \\
\hline Inglés & $84,5 \%$ & $90,5 \%$ & $95,9 \%$ \\
Francés & $3,8 \%$ & $1,9 \%$ & $1,0 \%$ \\
Alemán & $5,1 \%$ & $2,5 \%$ & $1,1 \%$ \\
Español & $0,7 \%$ & $0,4 \%$ & $0,3 \%$ \\
Japonés & $0,7 \%$ & $0,5 \%$ & $0,3 \%$ \\
N. ${ }^{0}$ total documentos & 554.598 & 689.629 & 956.533 \\
\hline
\end{tabular}

dente en las aportaciones de radiología a las revistas internacionales (etapa 1) que, sin embargo, no llega a concentrarse en las mejores revistas de la especialidad (etapa 2). La producción internacional de cardiología es menor, pero más estable y con un mayor porcentaje de documentos en las revistas de más prestigio de la especialidad. Es interesante señalar que, aunque estos resultados se refieren a un bajo número de documentos, un comportamiento similar se ha descrito para el área general de la radiología (alta tasa de incremento, tendencia a publicar en revistas del segundo cuartil) y de la cardiología (menor tasa de incremento, tendencia a publicar en revistas del primer cuartil) en España, a través del SCI en el período 1994-19994.

En segundo lugar, hay que resaltar el importante papel de la revista española de cada una de las sociedades científicas, es decir, Radiología y REvisTA ESPAÑOLA DE CARDIOLOGía, que publicaron la mitad de los documentos identificados en cada una de las dos especialidades. Dichas revistas tienen que asumir actualmente el reto de captar originales de calidad a pesar de la migración de los autores hacia revistas extranjeras. Es un hecho que el proceso de internacionalización ha puesto en peligro la supervivencia de algunas revistas nacionales, que han tenido que desarrollar distintas estrategias para resultar atractivas al investigador. Muchas revistas potencian su faceta internacional a través de distintas iniciativas, como son aumentar su presencia en bases de datos internacionales, estar disponibles en Internet u ofrecer sus contenidos en inglés.

El uso del inglés como idioma de publicación es una estrategia útil para aumentar la visibilidad internacional de las revistas pero, sobre todo, en las áreas más básicas o añadido a la edición en español en las áreas clínicas. Hoy por hoy, muchas revistas clínicas o aplicadas tienen su mayor audiencia en el propio país, en el que cumplen una importante función en la integración y cohesión de la comunidad científica nacional, contribuyendo a la práctica profesional y a la formación continuada, todo lo cual aconseja el uso de la lengua local. Así lo han entendido distintas revistas clínicas españolas que han decidido mantener su edición en español, a pesar de la creciente internacionalización de 
la ciencia, añadiendo en algunos casos una edición en inglés para aumentar su visibilidad fuera del país. Conseguir revistas atractivas tanto para el investigador especializado como para el clínico práctico es un reto importante que se plantean actualmente muchas revis$\operatorname{tas}^{7}$.

Como parte del proceso de internacionalización, el afán por publicar en las «mejores» revistas de la especialidad y vincular la calidad al factor de impacto de las revistas se ha extendido a todas las áreas. No obstante, algunos editores de revistas médicas nos recuerdan que, por encima de la obsesión por los factores de impacto, la revista más adecuada para publicar un documento dependerá de la audiencia a la que vaya dirigido $^{8}$. Curiosamente, publicar en las revistas con un alto factor de impacto recogidas en las principales bases de datos y escritas en inglés, es decir, en revistas de alto prestigio internacional, puede llegar a ser en algunos casos contraproducente si dichas revistas no son las que leen los investigadores a los que van dirigidos los documentos. De esta forma, se puede llegar a la paradójica situación de que estos trabajos apenas se difundan en el propio país, que puede ser donde en realidad están sus principales lectores potenciales y donde mayor utilidad pueden tener. Como resultado de su escasa difusión entre los que serían sus máximos lectores y posteriores citantes, los documentos pueden recibir menos citas y producir, en definitiva, menos impacto del que hubieran causado de haber sido publicados en la lengua natal. En concreto, documentos relativos a la medicina en un país pueden afectar más a la práctica e incluso a la gestión médica si se publican en la lengua del propio país.

Por otro lado, no se puede olvidar las limitaciones del factor de impacto, ampliamente recogidas en la bibliografía9. Aunque el factor de impacto nos permite identificar las revistas más prestigiosas de ámbito internacional, que suelen contar con una rigurosa selección de originales basada en criterios de calidad, no nos dice nada acerca del impacto real que produce luego cada documento, y se ha descrito una gran variabilidad entre documentos dentro de cada revista. Se puede acudir para ello a otros indicadores, como el número de citas recibidas por los documentos o el número de lecturas de los documentos electrónicos. Aparte de estos indicadores, y refiriéndonos en concreto a la investigación clínica, sería interesante valorar otros aspectos del impacto de la investigación como, por ejemplo, su influencia sobre la práctica clínica o su impacto social ${ }^{10}$.

A pesar de que los investigadores españoles cuentan hoy día con publicaciones en revistas de alta calidad y prestigio internacional, el reconocimiento posterior, medido a través de las citas, está todavía en muchas disciplinas por debajo de lo que sería esperable en función de la revista de publicación o por debajo del promedio mundial de la disciplina correspondiente. Así, atendiendo a los Science \& Technology Indicators norteamericanos de 2002, España producía en 1999 el 2,3\% de los documentos del SCI Expanded, pero sólo recibía el 1,6\% de las citas.

Podríamos entonces introducir una nueva cuarta etapa en el proceso de internacionalización, en la que la investigación de un país recibe un reconocimiento acorde con su capacidad, esfuerzo y contribución a la ciencia internacional. Y nuestro país tiene todavía un largo camino que recorrer, aunque algunos estudios ${ }^{11}$ muestran que, durante los últimos años, la visibilidad internacional y el impacto real de las publicaciones de autores españoles han mostrado una tendencia ascendente, medidos a través del número de citas por documento y del porcentaje de documentos citados.

\section{BIBLIOGRAFÍA}

1. Jiménez Contreras E, Moya Anegón F, Degado López-Cozar E. The evolution of research activity in Spain: the impact of the National Commission for the Evaluation for Research activity (CNAI). Res Policy 2003;32:123-42.

2. Zitt M, Perrot F, Barré R. The transition from «national» to «transnational» model and related measures of countries' performance. JASIS 1998;49:30-42.

3. Van Raan AFJ. Science as an international enterprise. Sci Public Policy 1997;24:290-300.

4. Gómez I, Fernández MT, Bordons M, Morillo F. La producción científica española en Medicina en los años 1994-1999. Rev Clin Esp 2004;204:75-88.

5. Miguel-Dasit A, Martí-Bonamatí L, Aleixandre R, Sanfeliu P, Valderrama JC. Producción española sobre diagnóstico por la imagen en cardiología y radiología (1994-1998). Rev Esp Cardiol 2004:57:806-14.

6. Bermejo J, Alfonso F, Bosch X. Técnicas de imagen en la medicina cardiovascular moderna. Rev Esp Cardiol 2003;56:193-4.

7. Bosch X, Villacastín JP, Alonso J. Revista Española de Cardiología en Journal Citation Reports. Rev Esp Cardiol 2000;53:1421-4.

8. Gastel B. Assessing the impact of investigators work; beyond impact factors. Can J Anaesth 2001;48:941-5.

9. Bordons M, Zulueta MA. Evaluación de la actividad científica a través de indicadores bibliométricos. Rev Esp Cardiol 1999; 52:790-800.

10. Van Diest PJ, Holzel H, Burnett D, Crocker J. Impactitis: new cures for an old disease. J Clin Pathol 2001;54:817-9.

11. Camí J, Suñen E, Carbó JM, Coma L. Producción científica española en Biomedicina y Ciencias de la Salud. Mapa bibliométrico de la investigación realizada en España durante el período 19942000. Informe para el Instituto de Salud Carlos III-Fondo de Investigación Sanitaria (FICV0077/02). Barcelona: Institut Municipal d'Investigació Mèdica, 2002. 